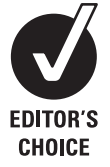

Imperial College Kidney and Transplant Institute, Hammersmith Hospital, London, UK

Correspondence to: Dr E Salisbury, Renal Department, 4th floor, Hamm House, Hammersmith Hospital, London W12 OHS, UK, emma.salisbury08@ imperial.ac.uk

Received 28 April 2008 Accepted 22 October 2008

\title{
Changing practice to improve pain control for renal patients
}

\author{
E M Salisbury, D S Game, I Al-Shakarchi, M Chan, L Fishman, L Tookman, E A Brown
}

\section{ABSTRACT}

Pain is a common symptom described by patients with end-stage kidney disease (ESKD) but remains ineffectively managed. The aim of this audit was to determine what proportion of these patients report pain, then introduce the use of an analgesic ladder adapted specifically for ESKD, and finally re-evaluate the prevalence of pain symptoms, looking for an improvement. A cohort of inpatients on the renal wards of a West London teaching hospital was studied. The number of patients reporting pain and the severity of their pain on a scale of 1-10 were recorded. A considerable number of patients were barred from participating because of a language barrier. Interpreters were introduced, and the phase was repeated. The World Health Organization (WHO) threestep analgesic ladder was adapted for patients with ESKD and introduced to medical staff on the renal wards. The number of patients reporting pain and the severity of their pain were re-recorded. There was a significant reduction in the number of patients reporting pain and the severity of their pain. Pain control in patients with ESKD is improved through the use of an adapted version of the WHO analgesic ladder. Strategies must be in place for effective communication with foreign patients.

Pain is one of the most common symptoms described by patients with end-stage kidney disease (ESKD), but it continues to be ineffectively managed. ${ }^{1-6}$ We believe that there are three key reasons for this. (1) There is a general lack of recognition of the problem, in part because of poor communication with patients. (2) The pathology of the pain is poorly understood and its nature is often chronic and complex. A renal patient may experience pain as a result of their primary disease (eg, polycystic kidneys), renal failure (eg, calciphylaxis), dialysis (eg, abdominal distension from peritoneal dialysis or steal syndrome with an arteriovenous fistula for haemodialysis) or a comorbidity (eg, peripheral vascular disease or diabetic neuropathy), and most likely there is more than one cause. (3) The pharmacokinetics and pharmacodynamics of many analgesic drugs are altered in patients with ESKD. Junior medical staff often lack the specialist knowledge required to adjust prescriptions. Their fear of precipitating an adverse effect leads to a reluctance to prescribe effective doses of analgesia.

This is a timely and universal clinical issue. Patients with ESKD will present to all specialties as a result of their multiple comorbidities, and the incidence and prevalence of chronic kidney disease are rising in Europe and the USA as a result of the increased prevalence of cardiovascular disease, diabetes, hypertension and obesity in the ageing population. There is also a significantly raised incidence of ESKD among the immigrant IndoAsian population.

The aim of this study was to improve pain management for renal patients at the West London Renal and Transplant Centre (WLRTC), using local audit and evaluation of current practice to raise awareness of the problem and instigate the introduction of new strategies for change and improvement.

\section{DESCRIPTION OF CONTEXT}

This study was conducted between August 2006 and April 2007 on the wards of the WLRTC, part of Imperial College Healthcare NHS Trust and situated at Hammersmith Hospital, Du Cane Road, London. The WLRTC has 76 general renal beds, split between three wards, and a 12-bed renal high-dependency unit. Each ward is managed dayto-day by one or two ST1/2 doctors (previously senior house officer), one StR doctor (previously specialist registrar) and a full complement of qualified nursing staff. A consultant nephrologist conducts a formal ward round twice daily. The ST1/2 and StR doctors are responsible for prescribing analgesia for the patients on the ward, and the nursing staff are responsible for its administration. A renal pharmacist screens each patient's drug chart on a daily basis and will optimise prescribing decisions. The pharmacists cannot prescribe analgesia.

\section{DESIGN}

Assessments of pain before and after the intervention were performed using standardised questionnaires, which simply recorded basic data (age, gender, ethnic origin) and the patient's perception of pain at the time of questioning on a scale up to 10. Questionnaires were administered to each patient by one of the authors over a 2 -week period.

\section{Participants}

To be eligible for participation in the study, patients had to be older than 18 years, possess decision-making capacity, and be not less than 7 days post surgery.

\section{STRATEGIES FOR CHANGE}

\section{Raising awareness of the problem: formal} presentation to multidisciplinary team

We presented details of the proposed study at a renal grand round to which all multidisciplinary staff (nursing staff, pharmacists, dieticians, physiotherapists, occupational therapists, counsellors) working in the renal unit were invited. The renal 
Box 1: Barriers to effective pain management for renal patients

1. Lack of recognition of the problem

2. Poor communication with patients

3. Lack of understanding

4. Altered pharmacokinetics, pharmacodynamics and adverse effects

5. Lack of training

grand round at the WLRTC occurs weekly at a fixed time and is both well publicised and well attended. (Attendance is obligatory for doctors in training, and clinical commitments are scheduled around the meeting.) Time is set aside for debate and discussion generated by the preceding presentation, and participation is positively encouraged from all members of the multidisciplinary team. This was an excellent forum in which to raise awareness of the problems of pain management for renal patients and develop initiatives for improvement.

Considerable work went into creating a high-quality PowerPoint presentation, which opened with a case report describing the inappropriate and inadequate pain management of an anonymous renal patient and then addressed what the authors believe to be the barriers to effective pain management for renal patients (box 1), supported by relevant literature and preliminary data collected at the WLRTC. A proposition for change was put to the audience, and further strategies evolved.

\section{Endorsing the use of interpreters}

In phase 1 of the study, 77 patients had met the inclusion criteria for participation, but 32 (42\%) did not speak English to a level that enabled the English-speaking investigator to approach them for participation. The importance of open communication and the establishment of a secure physician-patient relationship are well recognised in pain management, ${ }^{7}$ and these results highlight the presence of a vulnerable subset of patients in whom language can act as a barrier to optimal pain management and optimal medical care. The use of interpreters (Englishspeaking relatives, multilingual medical staff or members of the hospital interpreter service) was promoted and encouraged.

\section{Developing a renal adaptation of the World Health Organization (WHO) three-step analgesic ladder}

Although originally designed for cancer pain relief, the conceptual framework of the WHO analgesic ladder is now used throughout the specialties to prescribe analgesia in a logical stepwise approach, and its use is promoted from medical school onwards. Its advantages are its simplicity, applicability to a wide variety of situations and prescribers worldwide, and its safety. The concept of an analgesic ladder is robust and useful, particularly for junior or inexperienced prescribers, and its principles underlie good pain management in many situations.

The use of the WHO three-step analgesic ladder to treat patients with ESKD has been recommended in two review articles in nephrology literature, ${ }^{89}$ and is further supported by the recent prospective validation of Barakzoy and Moss, ${ }^{1}$ which achieved adequate analgesia in $96 \%$ of patients. However, it is inappropriate for use in patients with ESKD in its original form because non-steroidal anti-inflammatory drugs are usually contraindicated and adverse side effects remain a problem. In particular, the morphine metabolites morphine-3-glucuronide
Box 2: Time line illustrating study design and interventions

\author{
Phase 1 data collection \\ $\downarrow$ \\ Formal presentation to multidisciplinary team \\ $\downarrow 1$ month. Interpreters introduced \\ Phase 2 data collection \\ $\downarrow 3$ months. Analgesic ladder developed \\ Renal adaptation of WHO analgesic ladder introduced to all wards \\ $\downarrow 2$ months. Staff familiarise themselves with ladder \\ Phase 3 data collection
}

and morphine-6-glucuronide accumulate in renal failure and are not removed on dialysis. Therefore morphine is not recommended.

Discussion at the renal grand round prompted the decision to develop a renal adaptation of the WHO analgesic ladder, and one consultant nephrologist, one registrar and two senior renal pharmacists were appointed to draw up the renal adaptation of the analgesic ladder. Over the following weeks, they sought advice from other nephrologists, pharmacists, palliative care physicians and the pain control team. For high-strength opiate analgesia, fentanyl and alfentanil were felt to be most suitable because of high potency, quick onset of action, and much shorter half-life than morphine; they can be delivered subcutaneously or transdermally and are largely metabolised in the liver to inactive metabolites, so accumulation is not a problem. For oral high-strength opiates, oxycodone has the advantage of being well tolerated, mostly metabolised by the liver, and easily titratable. Anecdotally we have experienced few problems with respiratory suppression with oxycodone. For moderate-strength oral opiates, tramadol is well tolerated and effective at a reduced dose, perhaps because of its non-opiate mechanisms of action (eg, inhibition of norepinephrine and serotonin uptake). Similarly, oral codeine can be useful at modified dose, but its active metabolites (codeine-6-glucuronide and morphine) can accumulate, so patients should be monitored for recognised side effects.

The final draft was presented at the WLRTC consultant meeting and emailed to every member of the unit for feedback before finalisation.

\section{KEY MEASURES FOR IMPROVEMENT}

The number of patients reporting pain and the severity of their pain were compared before and after the intervention.

\section{Statistical analysis}

The normal approximation to the binomial distribution was used to test the null hypothesis-that an equal number of patients report pain before and after the intervention-against the alternative hypothesis - that fewer patients report pain after the intervention. $\mathrm{p}<0.05$ was considered significant.

The law of large numbers was used to determine an asymptotic distribution for the sample means of patients' pain scores out of 10 pre-intervention and post-intervention. The null hypothesis that there is no difference between patients' pain scores before and after the intervention was tested against the alternative hypothesis that patients' pain scores were lower after the intervention. $\mathrm{p}<0.05$ was considered significant. 
Table 1 Basic demographics describing the participants in each phase of the study

\begin{tabular}{|c|c|c|c|c|c|c|c|c|}
\hline & \multirow[b]{2}{*}{ No of participants } & \multirow[b]{2}{*}{ Mean age (years) } & \multicolumn{2}{|c|}{ Sex $(\%)$} & \multicolumn{4}{|c|}{ Ethnicity (\%) } \\
\hline & & & M & $\mathbf{F}$ & White & Asian & Black & Mixed \\
\hline Phase 1 & 45 & 58.9 & 60 & 40 & 58 & 11 & 22 & 9 \\
\hline Phase 2 & 52 & 59.6 & 60 & 40 & 52 & 23 & 17 & 8 \\
\hline Phase 3 & 58 & 61.5 & 64 & 36 & 45 & 33 & 21 & 1 \\
\hline
\end{tabular}

\section{EFFECTS OF CHANGE}

A total of 77 patients met the inclusion criteria in phase 1. Of these 77 patients, 32 (42\%) did not speak English to a level that enabled the English-speaking investigator to approach them for participation. The remaining $45(58 \%)$ patients agreed to participate and were interviewed. Table 1 shows basic demographics for each phase of the study.

Phase 2 was conducted 1 month after the grand round, and interpreters were used when necessary. Fifty-three patients met the inclusion criteria. One patient was excluded because of a language barrier. Twenty-eight (54\%) patients reported pain. The mean score for their pain was 5.64 out of 10 .

The renal adaptation of the WHO three-step analgesic ladder (fig 1) was introduced to the wards 2 months later, and phase 3 was conducted 2 months after that. Sixty patients met the inclusion criteria, and 58 (97\%) agreed to participate. Twentythree $(40 \%)$ reported pain (mean score 4.48 out of 10$)$.

Although patients may have participated in more than one phase, all were discharged and readmitted with different problems between phases of the study. No cases of opioid neurotoxicity or adverse effects were reported during the study.

In summary, the number of patients reporting pain after intervention was significantly reduced ( $p=0.0003$; fig $2 \mathrm{~A})$. The mean pain score was also significantly reduced, as depicted in fig $2 B$ for uncensored pain scores $(p=0.027)$ and fig $2 C$ with those patients reporting no pain censored out $(p=0.032)$.

\section{LESSONS LEARNT}

This study emphasises that pain is commonly experienced by patients with ESKD and it is under-treated. Through local audit and evaluation, it identifies five key barriers to effective pain management for renal patients (box 1), opening the way for change and improvement.

Positive departmental change is brought about through interprofessional discussion which raises awareness of the issue and prompts the introduction of interpreters to improve communication, and the development of a renal adaptation of the WHO analgesic ladder to address the problems of altered

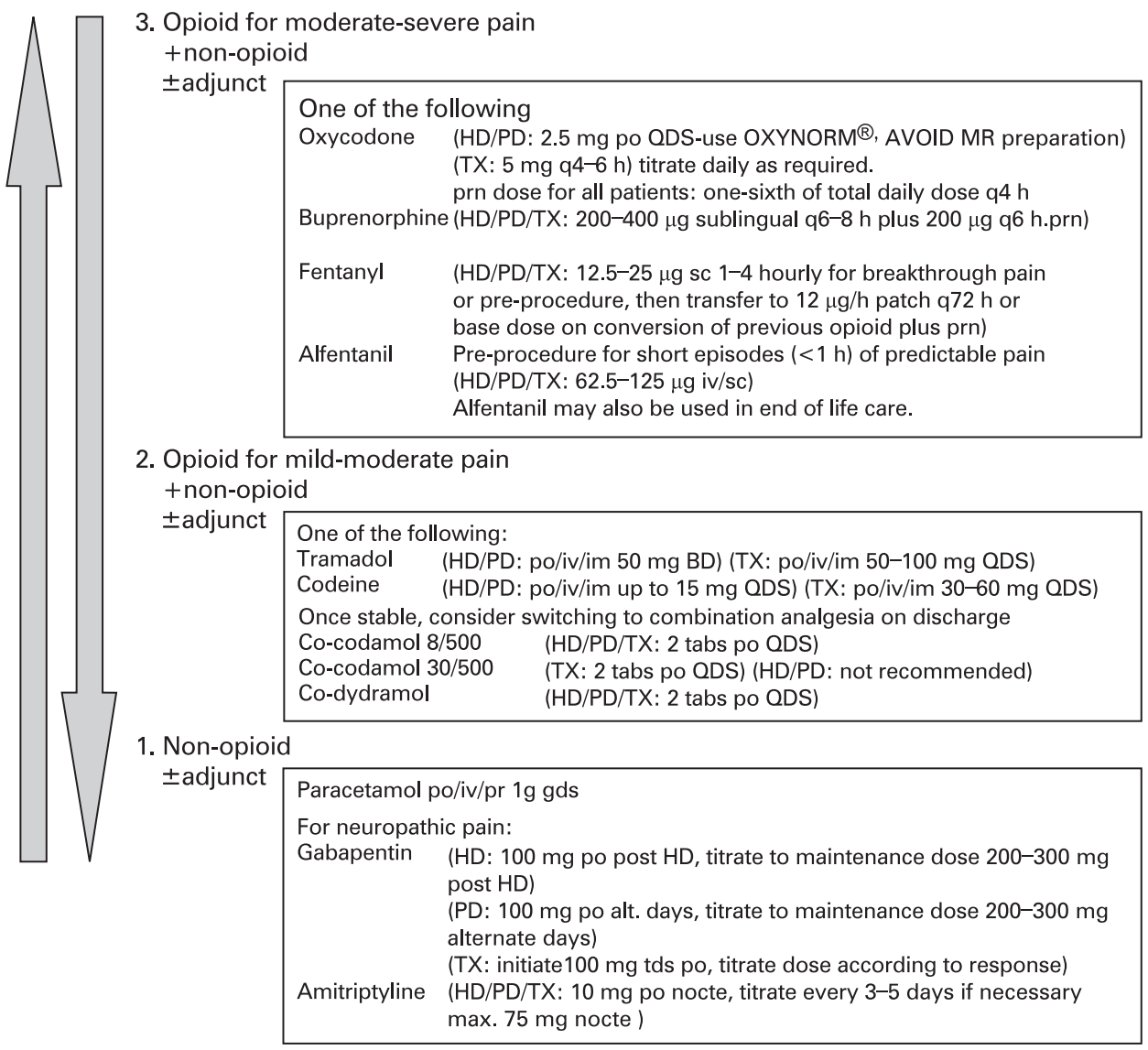

Figure 1 Renal adaptation of the WHO three-step analgesic ladder. gds, gradual dosage schedule; HD, haemodialysis; im, intramuscular; iv, intravenous; MR, modified release; PD, peritoneal dialysis; po, by mouth; prn, as needed; ODS, four times a day; sc, subcutaneous; tds, three times a day; TX, transplant. 

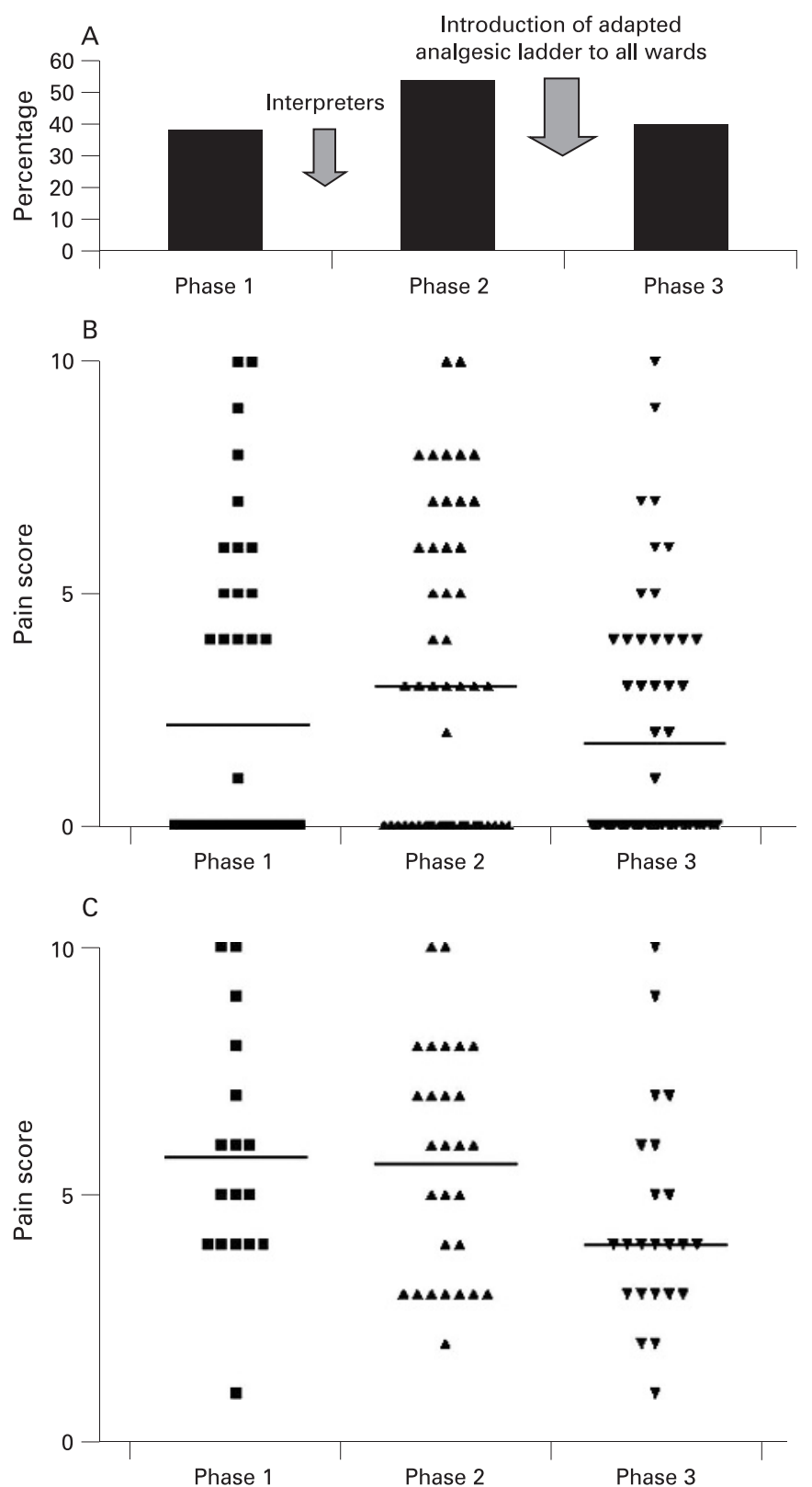

Figure 2 (A) Percentage of patients reporting pain at each phase. The use of interpreters increased the percentage reporting pain from 37.8 (phase 1) to 53.8 (phase 2); after the introduction of the adapted analgesic ladder the percentage fell to 39.7 (phase 3 ). The fall was significant $(p=0.0003)$. (B) Scatter of pain scores in each phase. The mean reported pain improved from 3.04 in phase 2 to 1.78 in phase 3 $(p=0.027)$. If those patients reporting no pain were censored out $(C)$, the mean pain score improved from 5.64 in phase 2 to 4.48 in phase 3 $(p=0.032)$.

pharmacology in patients with ESKD and lack of specialist training among junior medical staff responsible for prescribing analgesia.

Introduction of these changes led to a statistically significant reduction in the number of renal patients reporting pain and the severity of the pain they perceived. Barakzoy and Moss ${ }^{1}$ describe three cases of adverse drug effects when using the original WHO analgesic ladder in renal patients. No such cases were recorded using our adapted analgesic ladder.

\section{LIMITATIONS}

The results of this audit are unique to WLRTC and most probably to the time of the audit, but the strategies for change are universally applicable.

The study does not explore the ethnic difference in pain perception and the influence this may have had on results given the different sample makeup in each phase of the study. This is an area of potential further study relevant to units such as WLRTC with significant variation in patient ethnicity.

Lack of specialist knowledge among junior medical staff responsible for prescribing analgesia and reluctance to prescribe effective doses for fear of precipitating adverse effects were considered key factors in the poor pain management observed at WLRTC, but no assessment of doctors' experience of the renal adaptation of the WHO analgesic ladder was made. Had increased prescribing confidence among junior doctors been a key measure for improvement, a better appraisal of this study's contribution to professional development could have been made.

\section{CONCLUSION}

Batalden and Davidoff" define quality improvement as "the combined efforts of everyone-healthcare professionals, patients and their families, researchers, payers, planners, educators - to make changes that will lead to better patient outcomes (health), better system performance (care), and better professional development (learning)". This study is an excellent example.

Acknowledgements: EB, DG and ES were responsible for study design. ES, LF, LT and IA-S collected the data. DG and MC wrote the "renal adaptation of the world health organization three-step analgesic ladder" in consultation with a multidisciplinary team at Hammersmith Hospital. ES was responsible for data analysis, interpretation and writing the paper, with contributions from EB and DG. EB is guarantor.

Competing interests: None.

\section{REFERENCES}

1. Barakzoy AS, Moss AH. Efficacy of the World Health Organization analgesic ladder to treat pain in end-stage renal disease. J Am Soc Nephrol 2006;17:3198-203.

2. Weisbord SD, Fried LF, Arnold RM, et al. Prevalence, severity, importance of physical and emotional symptoms in chronic haemodialysis patients. J Am Soc Nephrol 2005;16:2487-94.

3. Moss AH. Improving end-of-life care for dialysis patients. Am J Kidney Dis 2005:45:209-12.

4. Baile GR, Mason NA, Bragg-Gresham JL, et al. Analgesic prescription patterns among haemodialysis patients in the DOPPS: potential for under prescription. Kidney Int 2004;65:2419-25.

5. Moss AH, Holley JL, Davison SN, et al. Core curriculum in nephrology: palliative care. Am J Kidney Dis 2004;43:172-3.

6. Davison SN. Pain in haemodialysis patients: prevalence, cause, severity, and management. Am J Kidney Dis 2003;42:1239-47.

7. Forde G, Stanos S. Practical management strategies for the chronic pain patient. J Fam Pract 2007;56:S13-22.

8. Kurella M, Bennett WM, Chertow GM. Analgesia in patients with ESRD: a review of available evidence. Am J Kidney Dis 2003:42:217-28.

9. Davison SN. Chronic pain in end-stage renal disease. Adv Chronic Kidney Dis 2005;12:326-34.

10. Batalden PB, Davidoff F. What is "quality improvement" and how can it transform healthcare? Qual Saf Health Care 2007:16:2-3 